\title{
OPTIMAL POSITIONS OF TUNABLE TRANSLATIONAL AND ROTATIONAL DYNAMIC ABSORBERS IN GLOBAL VIBRATION CONTROL IN BEAMS
}

\author{
WALDEMAR ŁATAS \\ Cracow University of Technology, Institute of Applied Mechanics, Kraków, Poland \\ e-mail: latas@mech.pk.edu.pl
}

\begin{abstract}
The paper discusses the problem of vibration of an Euler-Bernoulli beam with translational and rotational dynamic absorbers attached. The beam is subjected to concentrated and distributed harmonic forces. The equation of motion is solved using the Fourier method. The time Laplace transformation allows one to determine the amplitude-frequency characteristics of the beam deflection. The aim of the study is to examine the influence of positions of the translational and rotational dynamic absorbers on vibration suppression in the global control problem in beams. Numerical examples present reduction of kinetic energy of the cantilever beam with tunable absorbers over a wide range of frequencies. Optimal positions of the absorbers are obtained.
\end{abstract}

Keywords: dynamic vibration absorber, beam vibration, vibration reduction

\section{Introduction}

The main purpose of dynamic vibration absorbers (DVA) is suppression of motion at the point of attachment (Harris and Piersol, 2002; Korenev and Reznikov, 1993; Mead 1999). The most common DVAs attached to the vibrating structure subjected to harmonic excitation are passive tuned mass dampers (TMD). They are used to attenuate both the longitudinal and torsional vibration. Many works have been devoted to the optimization of dynamic absorbers parameters in both the linear and non-linear problems (Bisegna and Caruso, 2012; Krenk and Høgsberg, 2008; Lee et al., 2006; Mohtat and Dehghan-Niri, 2011; Rüdinger 2006; Sgobba and Marano, 2010; Tigli, 2012).

In civil engineering, TMDs are often used in structures susceptible to vibration induced by wind flow or seismic ground motion: suspension and cable-stayed bridges (Abdel-Rohman and Mariam, 2006; Chen and Cai, 2004; Chen and Wu, 2008), high-rise buildings (Bekdas and Nigdeli, 2011; Liu et al., 2008; Moon, 2011; Nagarajaiah and Varadarajan, 2005; Wang and Lin, 2007], chimneys (Ricciardelli, 2001; Brownjohn et al., 2010), masts, wind turbine towers (Eatas and Martynowicz, 2012). Tuned mass dampers are used in road and railway bridges (Li et al., 2005; Luu et al., 2012; Shi and Cai, 2008; Yau and Yang 2004a,b), footbridges (Caetano et al., 2010; Li et al., 2010) where the types of loading depend on the traffic of vehicles and pedestrians.

Because of multiple possible applications, a lot of attention has been put to the choice of parameters of dynamic absorbers in beams (Brennan and Dayou, 2000; Esmalizadeh and Jalili, 1998; Yang and Sedaghati, 2009; Younesian et al., 2006). In continuous structures such as beams, usually the best absorber locations are the points of loading application, but it may be technically difficult to do so. The problem is more difficult when non-collocated control and global vibration control are considered. In such cases or when the distributed loading is assumed, the main task is to find the appropriate positioning of damping devices on the structure (Brennan and Dayou, 2000; Cheung and Wong, 2008). 
The main drawbacks of passive TMDs are their limitations to narrow frequency bands only, ineffectiveness for non-stationary vibration and sensitivity for inaccurate tuning. To overcome these limitations, an active force is introduced between the damper and the structure (Lim, 2008; Wang and Lin, 2007). A disadvantage of active systems is, in turn, high energy consumption and dependence on the fault-free energy delivery. Their use can also cause instability of the structure. Semi-active systems which can alter stiffness or damping (Keye et al., 2009; Kim and Kang, 2012; Nagarajaiah and Varadarajan, 2005; Ricciardelli et al., 2000) in real time, improve the efficiency of passive systems, do not require large amounts of energy and cannot destabilize the system.

To improve the efficiency of vibration reduction, there are systems of mass dampers tuned to a single (Li and Ni, 2007; Li et al., 2005; Yau and Yang, 2004a,b) or several resonant frequencies for broadband excitation (Caetano et al., 2010; Luu et al., 2012). A special type of these systems are continuous absorbers (Thompson, 2008).

In this article, a model of the beam subjected to concentrated and distributed harmonic forces is given, with a system of translational and rotational dynamic vibration absorbers attached. The effect of rotational absorbers for the effectiveness of vibration reduction is investigated. Optimization of positions of the tunable absorbers in a cantilever beam subjected to a uniform distributed harmonic force with kinetic energy taken as the criterion is presented.

\section{Theoretical model}

Figure 1 presents a structure considered in the paper - a beam subjected to concentrated and distributed harmonic forces, with a system of translational and rotational dynamic vibration absorbers attached. The beam is of length $L$, with the following constant parameters: mass density $\rho$, cross-section area $A$, geometrical moment of inertia $I$, Young's modulus $E$.

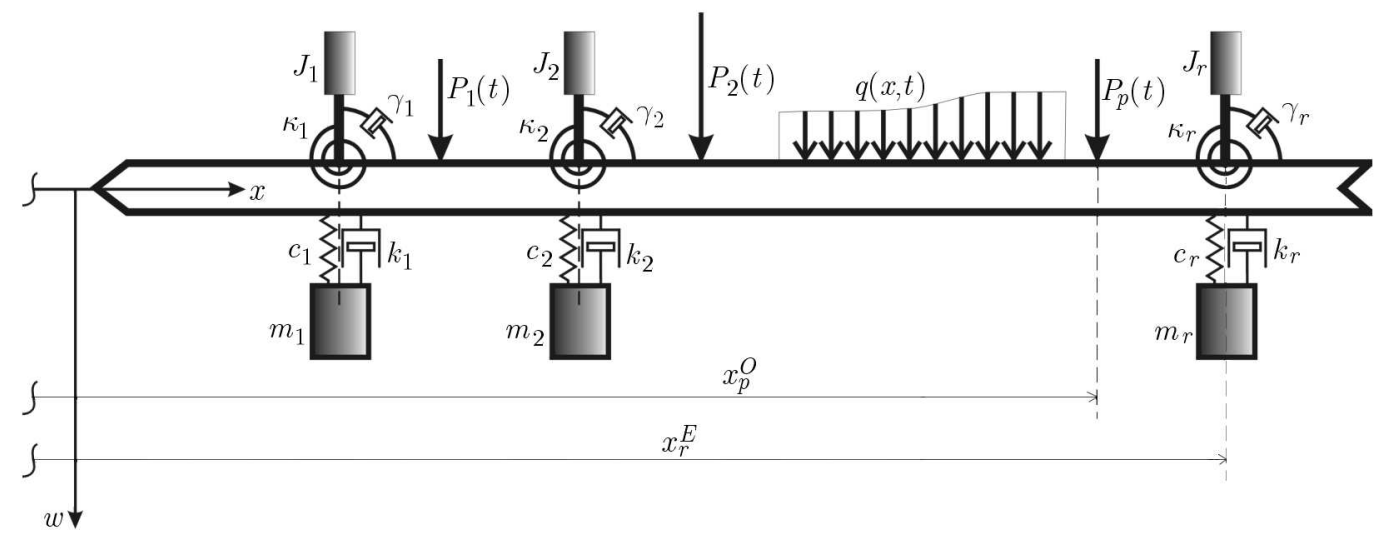

Fig. 1. Beam with a system of translational-rotational vibration absorbers

The equation of motion of the beam presented in Fig. 1 - assuming the Euler-Bernoulli theory and internal damping described by the Voigt-Kelvin rheological model (parameter $\alpha$ ) is given as (Cheung and Wong, 2008)

$$
\begin{gathered}
\rho A \frac{\partial^{2} w}{\partial t^{2}}+E I \alpha \frac{\partial^{5} w}{\partial x^{4} \partial t}+E I \frac{\partial^{4} w}{\partial x^{4}}=q(x, t)+\sum_{j=1}^{p} P_{j}(t) \delta\left(x-x_{j}^{O}\right) \\
+\sum_{j=1}^{r} F_{j}(t) \delta\left(x-x_{j}^{E}\right)+\sum_{j=1}^{r} \frac{\partial M_{j}(t) \delta\left(x-x_{j}^{E}\right)}{\partial x}
\end{gathered}
$$


where: $q(x, t)$ - distributed force; $P_{j}(t)$ - j-th concentrated force applied at the point $x_{j}^{O} ; F_{j}(t)-$ $j$-th concentrated force applied at the point $x_{j}^{E}$, acting on the beam from the translational dynamic vibration absorber; $M_{j}(t)-j$-th concentrated torque applied at the point $x_{j}^{E}$, acting on the beam from the rotational dynamic vibration absorber; $m_{j}, c_{j}, k_{j}$ - mass, damping and stiffness coefficients of the $j$-th translational dynamic vibration absorber; $J_{j}, \gamma_{j}, \kappa_{j}$ - moment of inertia, damping and stiffness coefficients of the $j$-th rotational dynamic vibration absorber; $p$-number of concentrated forces; $r$ - number of translational-rotational vibration absorbers.

The term translational-rotational absorber represents two dampers, one rotational and one translational, mounted in the same place.

Solution to equation (2.1) is assumed in form of the series

$$
w(x, t)=\sum_{i=1}^{\infty} q_{i}(t) \varphi_{i}(x)
$$

where $\varphi_{i}(x)$ are the modes of vibration of the beam without absorbers attached, which are dependent on the specific boundary conditions. The functions $q_{i}(t)$ are time-dependent generalized co-ordinates that should be determined. It is assumed that the distributed loading has the form: $q(x, t)=h(t) g(x)$.

Substituting series (2.4) into equation (2.1) and performing the time Laplace transformation (with initial conditions equal to zero) leads to

$$
\begin{gathered}
\sum_{i=1}^{\infty}\left[\rho A s^{2} Q_{i}(s)+E I \alpha \beta_{i}^{4} s Q_{i}(s)+E I \beta_{i}^{4} Q_{i}(s)-a_{i} H(s)-\sum_{j=1}^{p} d_{j i} P_{j}(s)\right. \\
\left.\quad-\sum_{j=1}^{r} b_{j i} F_{j}(s)-\sum_{j=1}^{r} e_{j i} M_{j}(s)\right] \varphi_{i}(x)=0
\end{gathered}
$$

Assuming orthogonality of the functions $\varphi_{i}(x)$ with the weight function $\eta(x)$, the following values of the coefficients appearing in equation (2.3) are obtained

$$
a_{i}=\frac{1}{K_{i}^{2}} \int_{0}^{l} g(x) \varphi_{i}(x) d x \quad d_{j i}=\frac{\varphi_{i}\left(x_{j}^{O}\right)}{K_{i}^{2}} \quad b_{j i}=\frac{\varphi_{i}\left(x_{j}^{E}\right)}{K_{i}^{2}} \quad e_{j i}=\frac{-\varphi_{i}^{\prime}\left(x_{j}^{E}\right)}{K_{i}^{2}}
$$

where: $K_{i}^{2}=\int_{0}^{L} \eta(x) \varphi_{i}^{2}(x) d x$, and additionally, in equation (2.3) the notation is introduced $\beta_{i}^{4}=[\rho A /(E I)] \omega_{i}^{2} ; \omega_{i}$ is the $i$-th natural frequency of the beam without the vibration absorbers attached and with the internal damping neglected $(\alpha=0)$. In equation (2.3), the symbols: $Q_{i}(s)$, $H(s), P_{j}(s), F_{j}(s), M_{j}(s)$ denote the Laplace transforms of the: $q_{i}(t), h(t), P_{j}(t), F_{j}(t), M_{j}(t)$, respectively.

Taking into account the linear independence of the functions $\varphi_{i}(x)$, from equation $(2.3)$ an expression for the Laplace transform $W(x, s)$ of the beam deflection $w(x, t)$ can be obtained

$$
W(x, s)=\sum_{i=1}^{\infty} \frac{a_{i} H(s)+\sum_{j=1}^{p} d_{j i} P_{j}(s)+\sum_{j=1}^{r} b_{j i} F_{j}(s)+\sum_{j=1}^{r} e_{j i} M_{j}(s)}{\rho A s^{2}+E I(1+\alpha s) \beta_{i}^{4}} \varphi_{i}(x)
$$

and its derivative with respect to $x$

$$
\frac{\partial W(x, s)}{\partial x}=\sum_{i=1}^{\infty} \frac{a_{i} H(s)+\sum_{j=1}^{p} d_{j i} P_{j}(s)+\sum_{j=1}^{r} b_{j i} F_{j}(s)+\sum_{j=1}^{r} e_{j i} M_{j}(s)}{\rho A s^{2}+E I(1+\alpha s) \beta_{i}^{4}} \varphi_{i}^{\prime}(x)
$$


The transforms of the force $F_{j}(s)$ and torque $M_{j}(s)$ acting on the beam from the $j$-th translational-rotational vibration absorber attached at the point $x_{j}^{E}$ are given by the expressions (Cheung and Wong, 2008)

$$
F_{j}(s)=-W\left(x_{j}^{E}, s\right) \frac{\left(c_{j} s+k_{j}\right) m_{j} s^{2}}{m_{j} s^{2}+c_{j} s+k_{j}} \quad M_{j}(s)=-\Theta\left(x_{j}^{E}, s\right) \frac{\left(\gamma_{j} s+\kappa_{j}\right) J_{j} s^{2}}{J_{j} s^{2}+\gamma_{j} s+\kappa_{j}}
$$

where the notation $\Theta(x, s)=-\partial W(x, s) / \partial x$ is introduced.

The transforms given by formulas (2.7) should be inserted into expressions (2.5) and (2.6). The resulting transforms of the beam deflection and slope should be satisfied at the points where the absorbers are attached to the beam. These conditions yield a system of linear algebraic equations to determine the unknowns $W\left(x_{k}^{E}, s\right)$ and $\Theta\left(x_{k}^{E}, s\right)(k=1,2, \ldots, r)$.

To simplify the expressions, the following notations are introduced

$$
\begin{array}{ll}
W\left(x_{j}^{E}, s\right)=W_{j} & \Theta\left(x_{j}^{E}, s\right)=\Theta_{j} \\
\varphi_{i}\left(x_{j}^{E}\right)=\varphi_{i j} & \varphi_{i}^{\prime}\left(x_{j}^{E}\right)=\varepsilon_{i j} \\
a_{i} H(s)+\sum_{j=1}^{p} d_{j i} P_{j}(s)=A_{i} & \rho A s^{2}+E I(1+\alpha s) \beta_{i}^{4}=B_{i} \\
\frac{\left(c_{j} s+k_{j}\right) m_{j} s^{2}}{m_{j} s^{2}+c_{j} s+k_{j}} b_{j i}=D_{j i} & \frac{\left(\gamma_{j} s+\kappa_{j}\right) J_{j} s^{2}}{J_{j} s^{2}+\gamma_{j} s+\kappa_{j}} e_{j i}=E_{j i}
\end{array}
$$

The system of $2 r$ linear equations for the unknowns $W_{k}, \Theta_{k}(k=1,2, \ldots, r)$ takes the form

$$
\begin{aligned}
& W_{k}\left(1+\sum_{i=1}^{\infty} D_{k i} \frac{\varphi_{i k}}{B_{i}}\right)+\sum_{j=1, j \neq k}^{r} \sum_{i=1}^{\infty} W_{j} D_{j i} \frac{\varphi_{i k}}{B_{i}}+\sum_{j=1}^{r} \sum_{i=1}^{\infty} \Theta_{j} E_{j i} \frac{\varphi_{i k}}{B_{i}}=\sum_{i=1}^{\infty} A_{i} \frac{\varphi_{i k}}{B_{i}} \\
& \Theta_{k}\left(\sum_{i=1}^{\infty} D_{k i} \frac{\varepsilon_{i k}}{B_{i}}-1\right)+\sum_{j=1, j \neq k}^{r} \sum_{i=1}^{\infty} \Theta_{j} E_{j i} \frac{\varepsilon_{i k}}{B_{i}}+\sum_{j=1}^{r} \sum_{i=1}^{\infty} W_{j} D_{j i} \frac{\varepsilon_{i k}}{B_{i}}=\sum_{i=1}^{\infty} A_{i} \frac{\varepsilon_{i k}}{B_{i}}
\end{aligned}
$$

Having solved the system (2.9), the transforms of the forces $F_{j}(s)$ and torques $M_{j}(s)$ may be calculated from expressions (2.7) and used to calculate from formulas (2.5) and (2.6) the transforms of the deflection and slope of the beam. Assuming steady-state vibration, after substituting $s=\mathrm{j} \omega(\mathrm{j}=\sqrt{-1})$, the expressions for the deflection and slope of the beam in the frequency domain may be obtained.

\section{Numerical results - optimization of position of the tunable vibration absorbers}

The numerical algorithm allows one to calculate in the $s$-domain the transforms of the beam deflection and slope for arbitrary boundary conditions. For a harmonic excitation, the amplitude-frequency characteristics of the bending moment, transverse force, time-averaged kinetic energy of the whole beam or its part may be further calculated.

\subsection{Optimization of the position of the tunable translational-rotational vibration absorber}

A cantilever steel beam is excited by a uniform distributed harmonic force (Fig. 2). The beam is of length $l=1.0 \mathrm{~m}$, mass density $\rho=7800 \mathrm{~kg} / \mathrm{m}^{3}$, Young's modulus $E=2.1 \cdot 10^{11} \mathrm{~N} / \mathrm{m}^{2}$, with rectangular cross-section of width $b=0.05 \mathrm{~m}$ and height $h=0.005 \mathrm{~m}$. The internal damping of the beam is neglected. There is only one translational-rotational absorber attached at the point $x_{1}^{E}$. The aim of the absorber is to attenuate vibration of the beam. The time-averaged kinetic energy of the whole beam is used as the global measure of vibration. 


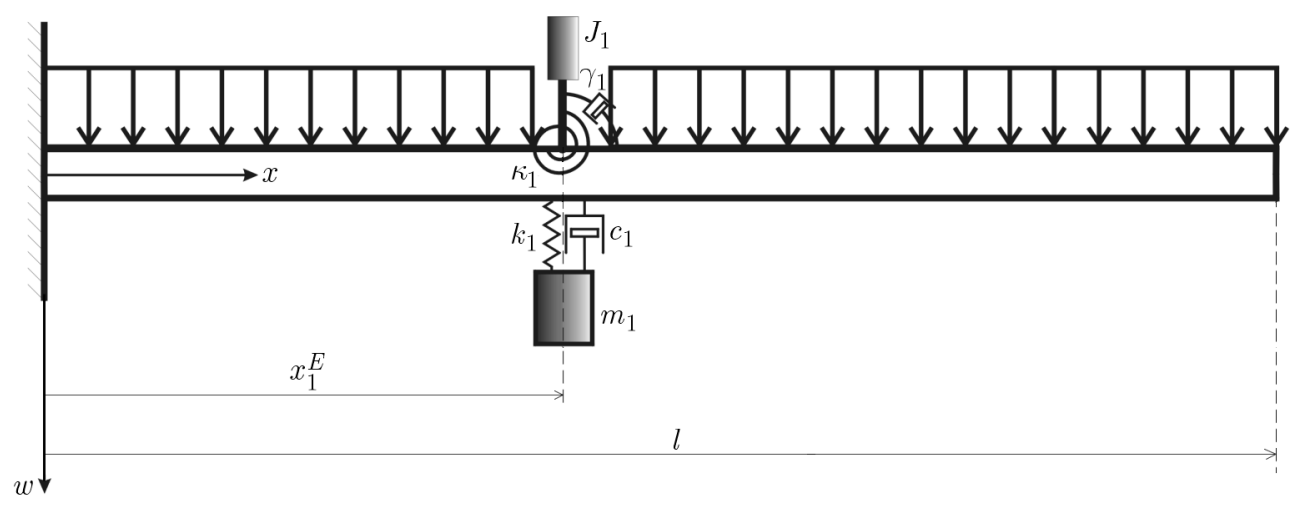

Fig. 2. Cantilever beam of length $l$ excited by a uniform distributed harmonic force with a dynamic translational-rotational vibration absorber attached

From the practical point of view, it is preferable to use tunable dampers (Brennan and Dayou, 2000; Dayou and Brennan, 2002) because a simple control algorithm can be used. It is assumed in further calculations that the absorbers attached are tuned so that they are resonant at each single frequency and do not have energy dissipating appliances $\left(c_{1}=0, \gamma_{1}=0\right)$. The first four natural frequencies of the presented beam are: $f_{1}=4.19 \mathrm{~Hz}, f_{2}=26.26 \mathrm{~Hz}, f_{3}=73.54 \mathrm{~Hz}$, $f_{4}=144.11 \mathrm{~Hz}$.

The main aim is to find the optimal position of the tunable translational-rotational vibration absorber for a given frequency band. For comparison, in Fig. 3 the calculated time-averaged kinetic energy for different positions of the single translational absorber $\left(J_{1}=0\right)$ in the range of frequency $\langle 0.0 \mathrm{~Hz}, 30.0 \mathrm{~Hz}\rangle$ is shown.

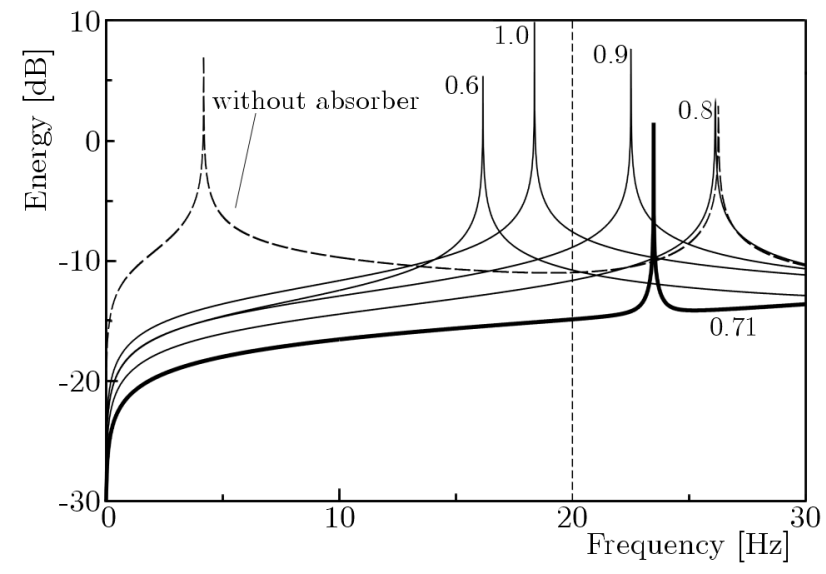

Fig. 3. Kinetic energy of the cantilever beam: without any absorber; with a single translational absorber placed at different points of the beam. The absorber attached is tuned to be resonant at each frequency. The numbers denote the dimensionless position of the absorber $x_{1}^{E} / l$

The best location of the single translational absorber in the range $\langle 0.0 \mathrm{~Hz}, 20.0 \mathrm{~Hz}\rangle$ is $x_{1 O P T}^{E}=0.71 l$.

For other locations, the results obtained are worse. The calculations performed for the translational-rotational absorber (translational and rotational absorbers are attached at the same location) give the same optimal position in the range $\langle 0.0 \mathrm{~Hz}, 40.0 \mathrm{~Hz}\rangle$ equal $x_{1 O P T}^{E}=0.71 l$. In Fig. 4, plots of kinetic energy calculated for the optimal locations of the translational and translational-rotational absorbers are shown for comparison.

The drawback of the tunable absorbers is that they cause an increase in the global vibration at the new natural frequencies of the resulting structure (Brennan and Dayou, 2000). It is clearly seen in Figs. 3 and 4. In the case of a single translational absorber, there is an additional 


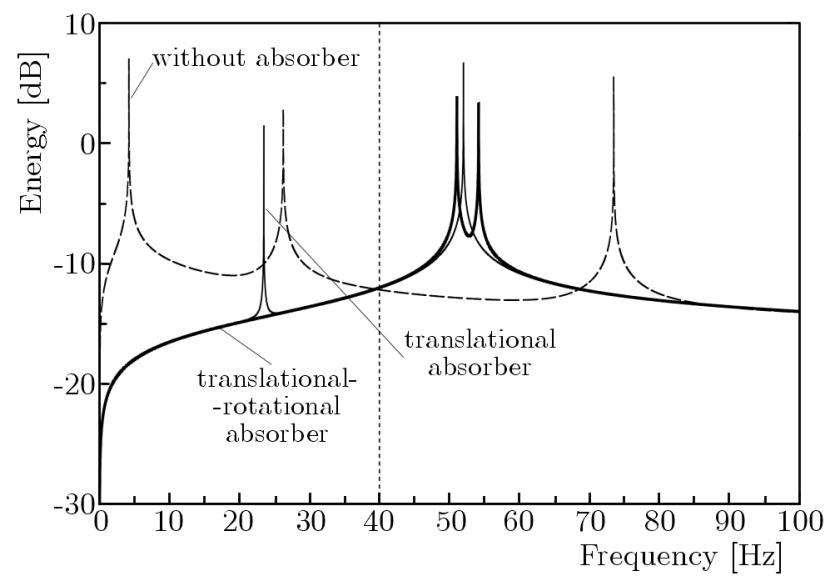

Fig. 4. Kinetic energy of the cantilever beam: without any absorber; with the translational and translational-rotational absorbers placed at the optimal position $x_{1 O P T}^{E}=0.71 l$. The absorbers attached are tuned to be resonant at each frequency

resonant frequency equal to $23.49 \mathrm{~Hz}$. The addition of the rotational absorber enhances vibration attenuation. There is no additional resonance at the frequency equal to $23.49 \mathrm{~Hz}$ and the translational-rotational absorber works efficiently up to frequency $40 \mathrm{~Hz}$.

In both cases, the optimal solutions are very sensitive to the accuracy of the absorber location. A relatively small change in it can significantly decrease the effectiveness of vibration suppression. A further improvement may be obtained by installing a few absorbers in different places of the beam.

In Sections 3.2 and 3.3 situations with two absorbers located at different places are presented. Referring to graphs in Fig. 4, the aim of optimization is to find the positions of the absorbers, which give the best vibration reduction efficiency in the given range $\langle 0.0 \mathrm{~Hz}, 40.0 \mathrm{~Hz}\rangle$.

\subsection{Optimization of positions of the tunable translational and rotational vibration absorbers}

Figure 5 presents the analyzed beam with two dynamic vibration absorbers without damping: one translational placed at the point $x_{1}^{E}$ and one rotational placed at the point $x_{2}^{E}$. The aim is to find the optimal locations of the absorbers $x_{1 O P T}^{* E}, x_{2 O P T}^{* E}$, which can give the best vibration reduction in the range $\langle 0.0 \mathrm{~Hz}, 40.0 \mathrm{~Hz}\rangle$. The absorbers attached are tuned to be resonant at each frequency. The results of numerical calculations are summarized in Section 3.4.

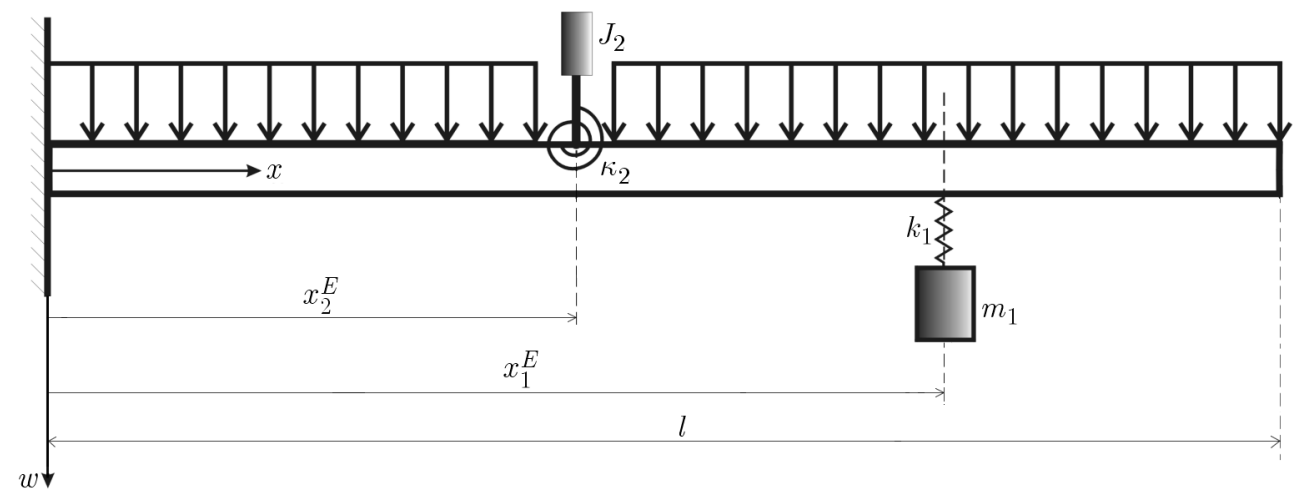

Fig. 5. Cantilever beam of length $l$ excited by a uniform distributed harmonic force with tunable dynamic translational and rotational vibration absorbers attached at different locations 


\subsection{Optimization of positions of the two tunable translational vibration absorbers}

Figure 6 presents the studied beam with two dynamic translational vibration absorbers without damping placed at the points $x_{1}^{E}, x_{2}^{E}$. The aim is to find the optimal locations of the absorbers $x_{1 O P T}^{* * E}, x_{2 O P T}^{* * E}$, which give the best vibration reduction in the range $\langle 0.0 \mathrm{~Hz}, 40.0 \mathrm{~Hz}\rangle$. The absorbers attached are tuned to be resonant at each frequency. The results of numerical calculations are gathered in Section 3.4.

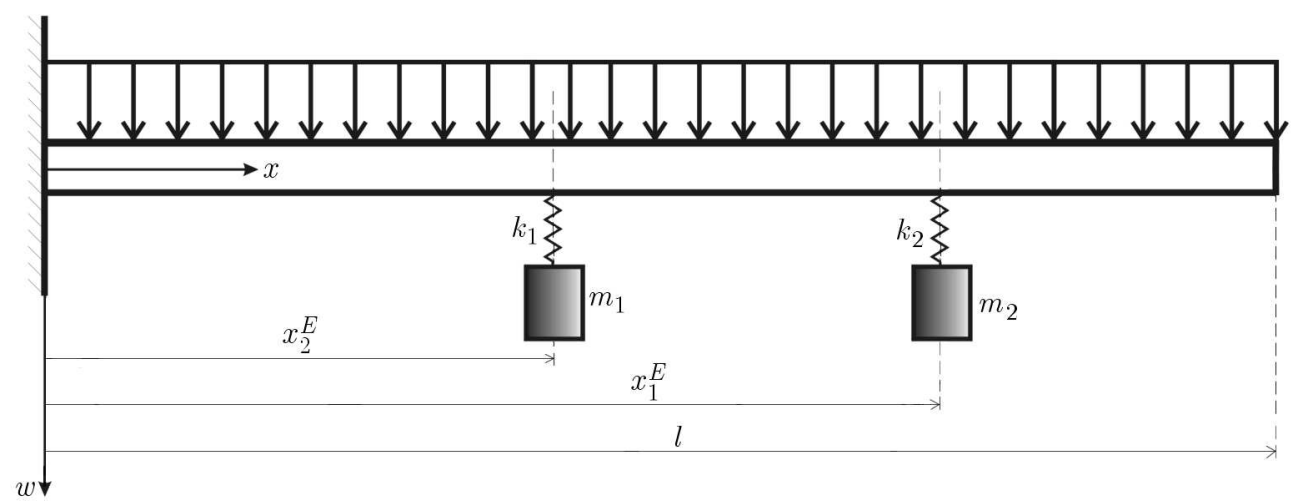

Fig. 6. Cantilever beam of length $l$ excited by a uniform distributed harmonic force with two tunable dynamic translational vibration absorbers attached

\subsection{Summary of the results of numerical calculations}

The calculated optimum positions of the absorbers for the cases presented in Section 3.2 and 3.3 are as follows:

- two absorbers; one translational of position $x_{1}^{E}$ and one rotational of position $x_{2}^{E}$ (Fig. 5)

$$
x_{1 O P T}^{* E}=0.60 l \quad x_{2 O P T}^{* E}=0.80 l
$$

- two translational absorbers of positions $x_{1}^{E}, x_{2}^{E}$ (Fig. 6)

$$
x_{1 O P T}^{* * E}=0.41 l \quad x_{2 O P T}^{* * E}=0.83 l
$$

In Fig. 7, plots of kinetic energy for the given above optimal positions of the absorbers in the two investigated cases are shown. For comparison, the kinetic energy for the optimal position of the translational-rotational absorber $\left(x_{1 O P T}^{E}=0.71 l\right.$, Fig. 2$)$ is also presented.

The option to use several absorbers in different locations greatly increases the efficiency of vibration suppression. The calculations performed show that the use of two translational absorbers gives a better result than the use of one translational and one rotational absorber. But if there is only one place to locate the absorber, the addition of the rotational absorber to the translational one may improve vibration attenuation.

The results presented are valid for a harmonic force uniformly distributed over the length of the beam. For another type of loading, the optimization can give different results, as for the other frequency bands. Further improvement of thr performance may be obtained by adding damping or de-tuning the absorbers (Brennan and Dayou, 2000).

\section{Conclusions}

The model presented in the paper can be used in local and global problems of optimal choice of positions and physical parameters of translational and rotational vibrations absorbers in beams. 


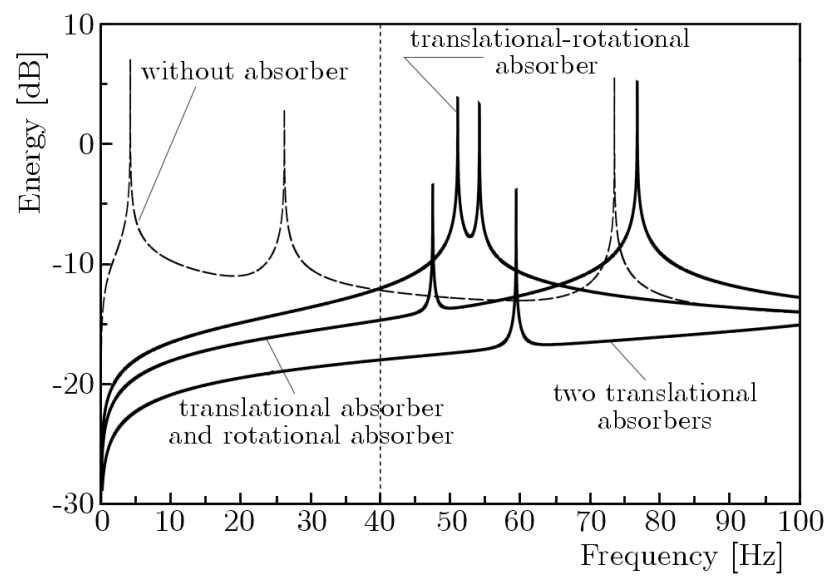

Fig. 7. Kinetic energy of the cantilever beam: without any absorber; with the translational-rotational absorber; with one translational and one rotational absorber; with two translational absorbers. The absorbers attached are placed at the optimal positions and are tuned to be resonant at each frequency

Theoretical calculations are illustrated by optimization of positions of the tunable absorbers in the global control of kinetic energy of the beam. The results of numerical calculations show that by adding a rotational absorber, the effectiveness of vibration reduction can be improved, as it can absorb rotational motion of the beam by applying torque to the beam. The addition of rotational absorbers to translational ones allows one to move resonances and expands the frequency band of effective vibration reduction. This effect can be also used in other structures such as frames, curved beams and pipes.

\section{References}

1. Abdel-Rohman M., Mariam J.J., 2006, Control of wind-induced nonlinear oscillations in suspension bridges using multiple semi-active tuned mass dampers, Journal of Vibration and Control, 12, $9,1011-1046$

2. Bekda s G., Nigdeli S.M., 2011, Estimating optimum parameters of tuned mass dampers using harmony search, Engineering Structures, 33, 2716-2723

3. Bisegna P., Caruso G., 2012, Closed-form formulas for the optimal pole-based design of tuned mass dampers, Journal of Sound and Vibration, 331, 2291-2314

4. Brennan M.J., Dayou J., 2000, Global control of vibration using a tunable vibration neutralizer, Journal of Sound and Vibration, 232, 3, 585-600

5. Brownjohn J.M.W., Carden E.P., Goddard C.R., Oudin G., 2010, Real-time performance monitoring of tuned mass damper system for a $183 \mathrm{~m}$ reinforced concrete chimney, Journal of Wind Engineering and Industrial Aerodynamics, 98, 169-179

6. Caetano E., Cunha Á., Magalhães F., Moutinho C., 2010, Studies for controlling humaninduced vibration of the Pedro e Inês footbridge, Portugal. Part 2: Implementation of tuned mass dampers, Engineering Structures, 32, 1082-1091

7. Chen S.R., Cai C.S., 2004, Coupled vibration control with tuned mass damper for long-span bridges, Journal of Sound and Vibration, 278, 449-459

8. Chen S.R., Wu J., 2008, Performance enhancement of bridge infrastructure systems: Long-span bridge, moving trucks and wind with tuned mass dampers, Engineering Structures, 30, 3316-3324

9. Cheung Y.L., Wong W.O., 2008, Isolation of bending vibration in a beam structure with a translational vibration absorber and a rotational vibration absorber, Journal of Vibration and Control, 14, 8, 1231-1246 
10. Dayou J., Brennan M.J., 2002, Global control of structural vibration using multiple-tuned tunable vibration neutralizers, Journal of Sound and Vibration, 258, 2, 345-357

11. Esmalizadeh E., Jalili N., 1998, Optimal design of vibration absorbers for structurally damped Timoshenko beams, ASME Journal of Vibration and Acoustics, 120, 833-841

12. Harris C.M., Piersol A.G., 2002, Harris' Shock and Vibration Handbook, McGraw-Hill

13. Keye S., Keimerb R., Homannc S., 2009, A vibration absorber with variable eigenfrequency for turboprop aircraft, Aerospace Science and Technology, 13, 165-171

14. Kim H.-S., Kang J.-W., 2012, Semi-active fuzzy control of a wind-excited tall building using multi-objective genetic algorithm, Engineering Structures, 41, 242-257

15. Korenev B.G., Reznikov L.M., 1993, Dynamic Vibration Absorbers, Theory and Technical Applications, Wiley, New York

16. Krenk S., HøgsberG J., 2008, Tuned mass absorbers on damped structures under random load, Probabilistic Engineering Mechanics, 23, 408-415

17. Lee Ch.-L., Chen Y.-T., Chung L.-L., Wangd Y.-P., 2006, Optimal design theories and applications of tuned mass dampers, Engineering Structures, 28, 43-53

18. Li H.-N., Ni X.-L., 2007, Optimization of non-uniformly distributed multiple tuned mass damper, Journal of Sound and Vibration, 308, 80-97

19. Li J., Su M., FAn L., 2005, Vibration control of railway bridges under high-speed trains using multiple tuned mass dampers, ASCE Journal of Bridge Engineering, 10, 3, 312-320

20. Li Quan., Fan J., Nie J., Li Quanwang., Chen Y., 2010, Crowd-induced random vibration of footbridge and vibration control using multiple tuned mass dampers, Journal of Sound and Vibration, 329, 4068-4092

21. Lim CH.-W., 2008, Active vibration control of the linear structure with an active mass damper applying robust saturation controller, Mechatronics, 18, 391-399

22. Liu M.-Y., Chiang W.-L., Hwang J.-H., Chu Ch.-R., 2008, Wind-induced vibration of highrise building with tuned mass damper including soil-structure interaction, Journal of Wind Engineering and Industrial Aerodynamics, 96, 1092-1102

23. Luu M., Zabel V., Knke C., 2012, An optimization method of multi-resonant response of highspeed train bridges using TMDs, Finite Elements in Analysis and Design, 53, 13-23

24. Eatas W., Martynowicz P., 2012, Modeling of vibration of wind turbine tower-nacelle system with dynamic absorber (in Polish), Modelowanie Inżynierskie, 44, 13, 187-198

25. Mead D.J., 1999, Passive Vibration Control, Wiley, New York

26. Mohtat A., Dehghan-Niri E., 2011, Generalized framework for robust design of tuned mass damper systems, Journal of Sound and Vibration, 330, 902-922

27. Moon K.S., 2011, Structural design of double skin facades as damping devices for tall buildings, Procedia Engineering, 14, 1351-1358

28. Nagarajaiah S., Varadarajan N., 2005, Short time Fourier transform algorithm for wind response control of buildings with variable stiffness TMD, Engineering Structures, 27, 431-441

29. Ricciardelli F., Occhiuzzi A., Clemente P., 2000, Semi active tuned mass damper control strategy for wind-excited structures, Journal of Wind Engineering and Industrial Aerodynamics, 87, $57-74$

30. Ricciardelli F., 2001, On the amount of tuned mass to be added for the reduction of the shedding-induced response of chimneys, Journal of Wind Engineering and Industrial Aerodynamics, 89, 1539-1551

31. RüDInger F., 2006, Tuned mass damper with fractional derivative damping, Engineering Structures, 28, 1774-1779 
32. Sgobba S., Marano G.C., 2010, Optimum design of linear tuned mass dampers for structures with nonlinear behaviour, Mechanical Systems and Signal Processing, 24, 1739-1755

33. Shi X., CAi C.S., 2008, Suppression of vehicle-induced bridge vibration using tuned mass damper, Journal of Vibration and Control, 14, 7, 1037-1054

34. Thompson D.J., 2008, A continuous damped vibration absorber to reduce broad-band wave propagation in beams, Journal of Sound and Vibration, 311, 824-842

35. Tigli O.F., 2012, Optimum vibration absorber (tuned mass damper) design for linear damped systems subjected to random loads, Journal of Sound and Vibration, 331, 3035-3049

36. WANG A.-P., Lin Y.-H., 2007, Vibration control of a tall building subjected to earthquake excitation, Journal of Sound and Vibration, 299, 757-773

37. Yang F., Sedaghati R., 2009, Vibration suppression of non-uniform curved beams under random loading using optimal tuned mass damper, Journal of Vibration and Control, 15, 2, 233-261

38. YAU J.-D., YANG Y.-B., 2004a, A wideband MTMD system for reducing the dynamic response of continuous truss bridges to moving train loads, Journal of Structural Engineering, 26, 1795-1807

39. YAU J.-D., YANG Y.-B., 2004b, Vibration reduction for cable-stayed bridges traveled by high-speed trains, Finite Elements in Analysis and Design, 40, 341-359

40. Younesian D., Esmailzadeh E., Sedaghati R., 2006, Passive vibration control of beams subjected to random excitations with peaked PSD, Journal of Vibration and Control, 12, 9, 941-953

Manuscript received May 9, 2014; accepted for print December 2, 2014 